\title{
Effect of T-channel distribution on firing pattern of the thalamocortical cell
}

\author{
Reza Zomorrodi ${ }^{* 1,2}$, Helmut Kroger ${ }^{1}$ and Igor Timofeev ${ }^{2}$
}

\author{
Address: ${ }^{1}$ Physics Department, Laval University, Quebec, Canada and ${ }^{2}$ Anatomy and Physiology Department, Laval University, Quebec, Canada \\ Email: Reza Zomorrodi* - rzomor@phy.ulaval.ca \\ * Corresponding author
}

from Sixteenth Annual Computational Neuroscience Meeting: CNS*2007

Toronto, Canada. 7-12 July 2007

Published: 6 July 2007

BMC Neuroscience 2007, 8(Suppl 2):PI 28 doi:I0.I I86/I47I-2202-8-S2-PI28

() 2007 Zomorrodi et al; licensee BioMed Central Ltd.

The low-threshold calcium current $\left(\mathrm{I}_{\mathrm{T}}\right)$ underlies burst generation in thalamocortical (TC) relay cells and plays a central role in the genesis of synchronized oscillations by thalamic circuits. Ascending and descending inputs to thalamic relay cells arrive on the dendritic tree, thus the study of synaptic integration in model cells requires simulations incorporating the electrically active properties of the dendritic tree. We developed a 3-compartment model of a thalamocortical cell to consider effects of dendritic currents on the response of the cell. First, we attribute uniform T-channel distribution for all compartments in the model, then we find a threshold value of channel permeability, $P_{c a}=1.56 \mathrm{e}-4(\mathrm{~cm} / \mathrm{sec})$ that enables generation of a low-threshold calcium spike (LTS). By multiplying the permeability of each section by its area we estimated the threshold number of channels necessary to reproduce an LTS. While we kept the total number of channels constant, we attributed different calcium permeability to the different compartments. Our simulations show, independent of the $\mathrm{Ca}^{2+}$ channel distribution, for a total channel number below the threshold value that the cell gives a passive response and above the threshold, the model reproduces the LTS spike. In a small range below the threshold the difference between uniform and non uniform distributions becomes visible. In the range of 1-2\% below threshold a uniform distribution of T-channels produces a passive response while a non-uniform distribution reproduces an LTS response. A comparison with experimental data of firing patterns and the I-V curve of the T-current shows a non-uniform distribution with higher density in sections near the soma better represents the experimental data. However, the geometry of the I-Vcurve of the T-current strongly depends on the quality of the voltage clamp. Depending on the electrode resistance, the maximum of the I-V-curve can change from $(6 \mathrm{nA},-60$ $\mathrm{mV})$ to $(3 \mathrm{nA},-40 \mathrm{mV})$. In addition we investigated the influence of the cell size on firing patterns. The model shows that with the same number of channels, the frequency of tonic firing increases with a decrease in the cell size, while the LTS response increases with an increase in cell size.

\section{Acknowledgements}

Supported by NSERC and CHIR. 